EPJ manuscript No.

(will be inserted by the editor)

\title{
Coulomb repulsion effects in driven electron transport
}

\author{
Franz J. Kaiser, Peter Hänggi, and Sigmund Kohler \\ Institut für Physik, Universität Augsburg, Universitätsstraße 1, D-86135 Augsburg, Germany
}

19 April 2007

\begin{abstract}
We study numerically the influence of strong Coulomb repulsion on the current through molecular wires that are driven by external electromagnetic fields. The molecule is described by a tight-binding model whose first and last site is coupled to a respective lead. The leads are eliminated within a perturbation theory yielding a master equation for the wire. The decomposition into a Floquet basis enables an efficient treatment of the driving field. For the electronic excitations in bridged molecular wires, we find that strong Coulomb repulsion significantly sharpens resonance peaks which broaden again with increasing temperature. By contrast, it has only a small influence on effects like non-adiabatic electron pumping and coherent current suppression.
\end{abstract}

PACS. 05.60.Gg Quantum transport - 85.65.+h Molecular electronic devices $-72.40 .+\mathrm{w}$ Photoconduction and photovoltaic effects - 73.63.-b Electronic transport in mesoscopic or nanoscale materials and structures

\section{Introduction}

Recent experiments on the conductance of single organic molecules opened a new direction in mesoscopic transport [1-3]. Of particular interest is thereby the influence of electronic and vibronic excitations of the molecules which leave their fingerprints in the resulting current-voltage characteristics. At low temperatures, these effects become more pronounced [4]. Much of our knowledge about excitations of molecules is based on spectroscopy, i.e. the optical response to light. In the context of molecular conduction, it has been proposed to study as well the signatures of such excitations in the transport quantifiers like the current [5-7] and its fluctuations [8]. Such experiments are at ' present attempted, but clearcut evidence for the proposed effects is still missing because the irradiation also causes unwanted thermal effects in the contacts, which in today's setups seem to dominate. One possibility to protect the contacts against the radiation is using the evanescent light at the tip of a near-field optical microscope.

Coupled quantum dots represent a setup with properties similar to those of molecular wires $[9,10]$, albeit at different length and energy scales. As compared to molecular wires, they are more stable and tunable, but have the disadvantage that only a few dots can be coupled coherently. The transport properties of these "artificial molecules" can be significantly modified by microwaves [10]. It has for example been demonstrated experimentally that resonant excitations between the levels of double quantum dots result in the so-called photon-assisted transport, i.e. a significant enhancement of the dc current $[11,12]$.

In addition to photon-assisted transport [13], other less intuitive phenomena have been predicted in this context. For example the so-called coherent suppression of tun- nelling in a double-well potential due to a time-dependent dipole force can also be found in ac driven transport through coupled quantum dots: For characteristic ratios between the amplitude and the frequency of the driving, it has been predicted that the dc current [14-16] and the shot noise [17] will be suppressed. A further prominent effect is adiabatic electron pumping, which is the generation of a dc current by means of a periodic variation of the conductor parameters in the absence of any net bias [18-21]. It has been proposed $[22,23]$ and experimentally demonstrated $[10,11]$ that pumping is more effective at internal resonances, i.e., beyond the adiabatic limit where, in addition, the pump current possesses a surprisingly low noise level [24].

Periodically time-dependent quantum systems can be described very efficiently within a Floquet theory which originally has been derived for driven closed quantum systems [25] and later been generalised to dissipative quantum systems [26,27]. Furthermore, it is possible to derive Floquet theories for the description of transport through mesoscopic conductors which are connected to external leads. For cases in which electron-electron interactions do not play any role, one can derive a Floquet scattering theory that provides exact expressions for the current $[17,28]$ and its noise $[8,17]$. Treating the coupling between the conductor and the leads perturbatively, one can obtain a master equation for the reduced density operator of the wire. This enables a rather efficient treatment of time-dependent transport after decomposing the wire density operator into a Floquet basis. Then it is possible to study relatively large driven conductors [29] and to include also electron-electron [30] and electron-phonon interactions [31]. If the time-dependent field consists of 


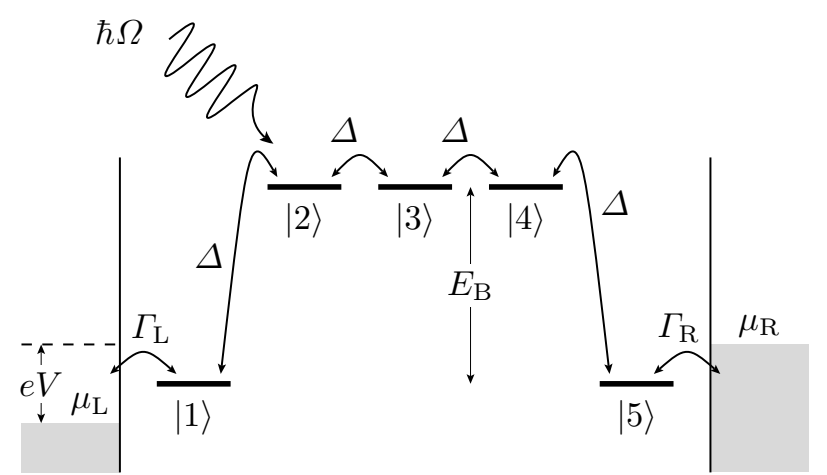

Fig. 1. Bridged molecular wire model consisting of $N=5$ sites with internal tunnelling matrix elements $\Delta$ and effective wire-lead coupling strengths $\Gamma_{\mathrm{L} / \mathrm{R}}$.

one or a few laser pulses, it is possible to obtain the density operator by propagating the Liouville-von Neumann equation [32].

In this work, we derive in Sections 2 and 3 a Floquet master equation formalism that captures situations in which strong Coulomb repulsion restricts the excess charge residing on the conductor to a single electron. For later reference, we adapt in Section 4 our approach to the case of spinless electrons and non-interacting electrons. We then investigate in Section 5 the role of strong Coulomb repulsion for photon-assisted transport through bridged molecular wires, non-adiabatic electron pumping, and coherent current suppression.

\section{The wire-lead model}

The system of the driven wire, the leads, and the coupling between the molecule and the leads, as sketched in Fig. 1, is described by the Hamiltonian

$$
\mathcal{H}(t)=\mathcal{H}_{\text {wire }}(t)+\mathcal{H}_{\text {leads }}+\mathcal{H}_{\text {wire-leads }} .
$$

The wire is modelled within a tight-binding description by the molecular orbitals $|n\rangle, n=1, \ldots, N$, so that

$$
\mathcal{H}_{\text {wire }}(t)=\sum_{n, n^{\prime}, s} H_{n n^{\prime}}(t) c_{n s}^{\dagger} c_{n^{\prime} s}+\frac{U}{2} N_{\text {wire }}\left(N_{\text {wire }}-1\right)
$$

where $c_{n s}^{\dagger}\left(c_{n s}\right)$ creates (annihilates) an electron with spin $s$ at site $|n\rangle$ and $\left[c_{n s}, c_{n^{\prime} s^{\prime}}^{\dagger}\right]_{+}=\delta_{n, n^{\prime}} \delta_{s, s^{\prime}}$. The influence of a driving field entails a $\mathcal{T}$-periodic time-dependence on the single-particle Hamiltonian $H_{n n^{\prime}}(t)=H_{n n^{\prime}}(t+\mathcal{T})$. The last term in Eq. (2) captures the electron-electron interaction within a capacitor model and the operator $N_{\text {wire }}=\sum_{n, s} c_{n s}^{\dagger} c_{n s}$ describes the number of excess electrons residing on the molecule. Below we shall assume that $U$ is so large that only states with zero or one excess electron play a role.

The first and the last site of the molecule, $|1\rangle$ and $|N\rangle$, couple via the tunnelling Hamiltonian

$$
\mathcal{H}_{\text {wire-leads }}=\sum_{q, s}\left(V_{\mathrm{L} q} c_{\mathrm{L} q s}^{\dagger} c_{1 s}+V_{\mathrm{R} q} c_{\mathrm{R} q s}^{\dagger} c_{N s}\right)+\text { H.c. }
$$

to the respective lead. The operator $c_{\mathrm{L} q s}^{\dagger}\left(c_{\mathrm{R} q s}^{\dagger}\right)$ creates an electron in the left (right) lead in the state $|\mathrm{L} q s\rangle$ which is orthogonal to all wire states. It will turn out that the influence of the tunnelling Hamiltonian is fully characterised by the spectral density

$$
\Gamma_{\ell}(\epsilon)=2 \pi \sum_{q}\left|V_{\ell q}\right|^{2} \delta\left(\epsilon-\epsilon_{q}\right)
$$

which becomes a continuous function of the energy $\epsilon$ if the lead states are dense. If all relevant lead states are located in the centre of the conduction band, the energydependence of the spectral densities is not relevant so that they can be replaced by a constant, $\Gamma_{\ell}(\epsilon)=\Gamma_{\ell}$. This defines the so-called wide-band limit.

The leads are modelled as ideal Fermi gases

$$
\mathcal{H}_{\text {leads }}=\sum_{q, s}\left(\epsilon_{q} c_{\mathrm{L} q s}^{\dagger} c_{\mathrm{L} q s}+\epsilon_{q} c_{\mathrm{R} q s}^{\dagger} c_{\mathrm{R} q s}\right)
$$

which are initially at thermal equilibrium with the chemical potential $\mu_{\mathrm{L} / \mathrm{R}}$ and, thus, are described by the density operator

$$
\rho_{\text {leads }, \text { eq }} \propto \exp \left[-\left(\mathcal{H}_{\text {leads }}-\mu_{\mathrm{L}} N_{\mathrm{L}}-\mu_{\mathrm{R}} N_{\mathrm{R}}\right) / k_{\mathrm{B}} T\right],
$$

where $N_{\ell}=\sum_{q} c_{q \ell}^{\dagger} c_{q \ell}$ denotes the electron number in lead $\ell=\mathrm{L}, \mathrm{R}$. Then all lead properties can be expressed in terms of the expectation value

$$
\left\langle c_{\ell q s}^{\dagger} c_{\ell^{\prime} q^{\prime} s^{\prime}}\right\rangle=\delta_{\ell \ell^{\prime}} \delta_{q q^{\prime}} \delta_{s s^{\prime}} f_{\ell}\left(\epsilon_{q}\right)
$$

where $f_{\ell}(\epsilon)=\left(1+\mathrm{e}^{\left(\epsilon-\mu_{\ell}\right) / k_{\mathrm{B}} T}\right)^{-1}$ denotes the Fermi function. Since a typical metal screens all electric fields with a frequency below the plasma frequency, we assume that the bulk properties of the leads are not affected by the laser irradiation.

\section{Master equation approach for strong Coulomb repulsion}

\subsection{Perturbation theory}

Most master equation approaches to electron transport [30-34] are based on a perturbative treatment of the wirelead Hamiltonian $H_{\text {wire-leads. }}$ Within these approaches, it is possible to include within a Floquet theory the response to a time-periodic field exactly $[8,35]$. The derivation of a master equation starts from the Liouville-von Neumann equation $\mathrm{i} \hbar \dot{\rho}(t)=[H(t), \rho(t)]$ for the total density operator $\rho(t)$ for which one obtains by standard techniques the approximate equation of motion

$$
\begin{aligned}
\dot{\rho}(t)= & -\frac{\mathrm{i}}{\hbar}\left[H_{\text {wire }}(t)+H_{\text {leads }}, \rho(t)\right] \\
& -\frac{1}{\hbar^{2}} \int_{0}^{\infty} \mathrm{d} \tau\left[H_{\text {wire-leads }},\left[\widetilde{H}_{\text {wire-leads }}(t-\tau, t), \rho(t)\right]\right]
\end{aligned}
$$


Franz J. Kaiser et al.: Coulomb repulsion effects in driven electron transport

Here the first term corresponds to the coherent dynamics of both the wire electrons and the lead electrons, while the second term describes resonant electron tunnelling between the leads and the wire. The tilde denotes operators in the interaction picture with respect to the molecule and the lead Hamiltonian without the molecule-lead coupling, $\widetilde{X}\left(t, t^{\prime}\right)=U_{0}^{\dagger}\left(t, t^{\prime}\right) X U_{0}\left(t, t^{\prime}\right)$, where $U_{0}$ is the propagator without the coupling. The net (incoming minus outgoing) electrical current through the left contact is given by minus the time-derivative of the electron number in the left lead multiplied by the electron charge $-e$. From Eq. (8) follows for the current in the wide-band limit the expression

$$
\begin{aligned}
I_{\mathrm{L}}(t)= & e \operatorname{tr}\left[\dot{\rho}(t) N_{\mathrm{L}}\right] \\
= & -e \frac{\Gamma_{\mathrm{L}}}{\pi \hbar} \operatorname{Re} \int_{0}^{\infty} \mathrm{d} \tau \int \mathrm{d} \epsilon \mathrm{e}^{\mathrm{i} \epsilon \tau / \hbar} \\
& \times\left\{\left\langle c_{1}^{\dagger} \tilde{c}_{1}(t, t-\tau)\right\rangle \bar{f}_{\mathrm{L}}(\epsilon)-\left\langle\tilde{c}_{1}(t, t-\tau) c_{1}^{\dagger}\right\rangle f_{\mathrm{L}}(\epsilon)\right\},
\end{aligned}
$$

where $\bar{f}_{\ell}=1-f_{\ell}$ and $\langle\cdots\rangle=\operatorname{tr}_{\text {wire }} \rho_{\text {wire }} \cdots$ denotes the average with respect to the wire density operator, which still has to be determined. We emphasise that the expectation values in Eq. (9) depend directly on the dynamics of the isolated wire and are thus influenced by the driving.

\subsection{Floquet theory}

An important feature of the current formula (9) is its dependence on solely the wire operators while all lead operators have been eliminated. Therefore it is sufficient to consider the reduced density operator $\rho_{\text {wire }}=\operatorname{tr}_{\text {leads }} \rho$ of the wire electrons. However, $\rho_{\text {wire }}$ still obeys a time-dependent master equation whose direct solution requires quite some effort, in particular if one is interested in the behaviour at long times. This effort can be reduced significantly by exploiting the fact that the master equation (8) inherited from the total Hamiltonian $\mathcal{H}(t)$ a $\mathcal{T}$-periodic timedependence, which opens the way for a Floquet treatment.

\subsubsection{Fermionic Floquet operators}

One possibility for such a treatment is to use the Floquet states of the central system, i.e. the driven wire, as a basis. Thereby we also use the fact that in the wire Hamiltonian (2), the single-particle contribution commutes with the interaction term and, thus, these two Hamiltonians possess a complete set of common eigenstates. Here we start by diagonalising the first part of the Hamiltonian which describes the single-particle dynamics determined by the time-periodic matrix elements $H_{n n^{\prime}}(t)$. According to the Floquet theorem, the corresponding (single particle) Schrödinger equation possesses a complete solution of the form

$$
\left|\Psi_{\alpha}(t)\right\rangle=\mathrm{e}^{\mathrm{i} \epsilon_{\alpha} t / \hbar}\left|\varphi_{\alpha}(t)\right\rangle,
$$

with the so-called quasienergies $\epsilon_{\alpha}$ and the $\mathcal{T}$-periodic Floquet states

$$
\left|\varphi_{\alpha}(t)\right\rangle=\sum_{k} \mathrm{e}^{-\mathrm{i} k \Omega t}\left|\varphi_{\alpha, k}\right\rangle
$$

The Floquet states and eigenenergies are obtained by solving the eigenvalue problem

$$
\left(\sum_{n, n^{\prime}}|n\rangle H_{n n^{\prime}}(t)\left\langle n^{\prime}\right|-\mathrm{i} \hbar \frac{\mathrm{d}}{\mathrm{d} t}\right)\left|\varphi_{\alpha}(t)\right\rangle=\epsilon_{\alpha}\left|\varphi_{\alpha}(t)\right\rangle,
$$

whose solution allows one to construct via Slater determinants many-particle Floquet states. In analogy to the quasimomenta in Bloch theory for spatially periodic potentials, the quasienergies $\epsilon_{\alpha}$ come in classes

$$
\epsilon_{\alpha, k}=\epsilon_{\alpha}+k \hbar \Omega, \quad k \in \mathbb{Z}
$$

of which all members represent the same physical solution of the Schrödinger equation. Thus we can restrict ourselves to states within one Brillouin zone like for example $0 \leq$ $\epsilon_{\alpha}<\hbar \Omega$.

For the computation of the current it is convenient to have an explicit expression for the interaction picture representation of the wire operators. It can be obtained from the (fermionic) Floquet creation and annihilation operators [36] defined via the transformation

$$
c_{\alpha s}(t)=\sum_{n}\left\langle\varphi_{\alpha}(t) \mid n\right\rangle c_{n s} .
$$

The inverse transformation

$$
c_{n s}=\sum_{\alpha}\left\langle n \mid \varphi_{\alpha}(t)\right\rangle c_{\alpha s}(t)
$$

follows from the mutual orthogonality and the completeness of the Floquet states at equal times [27]. Note that the right-hand side of Eq. (15) becomes time independent after the summation.

The Floquet annihilation operator (14) has the interaction picture representation

$$
\begin{aligned}
\tilde{c}_{\alpha s}\left(t, t^{\prime}\right) & =U_{0}^{\dagger}\left(t, t^{\prime}\right) c_{\alpha s}(t) U_{0}\left(t, t^{\prime}\right) \\
& =\mathrm{e}^{-\mathrm{i}\left(\epsilon_{\alpha}+U N_{\text {wire }}\right)\left(t-t^{\prime}\right) / \hbar} c_{\alpha s}\left(t^{\prime}\right),
\end{aligned}
$$

with the important feature that the time difference $t-t^{\prime}$ enters only via the exponential prefactor. This will allow us to evaluate the $\tau$-integration of the master equation (8) after a Floquet decomposition. Relation (17) can easily be shown by computing the time derivative with respect to $t$ which by use of the Floquet equation (12) becomes

$$
\frac{\mathrm{d}}{\mathrm{d} t} \tilde{c}_{\alpha s}\left(t, t^{\prime}\right)=-\frac{\mathrm{i}}{\hbar}\left(\epsilon_{\alpha}+U N_{\text {wire }}\right) \tilde{c}_{\alpha s}\left(t, t^{\prime}\right) .
$$

Together with the initial condition $\tilde{c}_{\alpha}\left(t^{\prime}, t^{\prime}\right)=c_{\alpha}\left(t^{\prime}\right)$ follows relation (17). Note that the time evolution induced by $\mathcal{H}_{\text {wire }}(t)$ conserves the number of electrons on the wire.

\subsubsection{Master equation and current formula}

In order to make use of the Floquet ansatz, we decompose the master equation (8) and the current formula (9) into the Floquet basis derived in the last subsection. For that 
purpose we use the fact that we are finally interested in the current at asymptotically large times in the limit of a large interaction $U$. The latter has the consequence that only wire states with at most one excess electron play a role, so that the density operator $\rho_{\text {wire }}$ can be decomposed into the $2 N+1$ dimensional basis $\left\{|0\rangle, c_{\alpha s}^{\dagger}(t)|0\rangle\right\}$, where $|0\rangle$ denotes the wire state in the absence of excess electrons and $s=\uparrow, \downarrow$. Moreover, it can be shown that at large times, the density operator of the wire becomes diagonal in the electron number $N_{\text {wire }}$. Therefore a proper ansatz reads

$$
\rho_{\text {wire }}(t)=|0\rangle \rho_{00}(t)\left\langle 0\left|+\sum_{\alpha, \beta, s, s^{\prime}} c_{\alpha s}^{\dagger}\right| 0\right\rangle \rho_{\alpha s, \beta s^{\prime}}(t)\langle 0| c_{\beta s^{\prime}}
$$

Note that we keep terms with $\alpha \neq \beta$, which means that we work beyond a rotating-wave approximation. Indeed in a non-equilibrium situation, the off-diagonal density matrix elements $\rho_{\alpha \beta}$ will not vanish and neglecting them might lead to artefacts $[8,37]$. In the context of molecular wires, such a treatment of strong Coulomb repulsion by a restriction to at most one excess electron has recently also been applied to incoherent [38-40] as well as to coherent transport [41].

By inserting the decomposition (19) into the master equation (8), we obtain an equation of motion for the matrix elements $\rho_{\alpha s, \beta s^{\prime}}=\left\langle 0\left|c_{\alpha s} \rho_{\text {wire }} c_{\beta s^{\prime}}^{\dagger}\right| 0\right\rangle$. We evaluate the trace over the lead states and compute the matrix element $\left\langle 0\left|c_{\alpha s}(t) \ldots c_{\beta s^{\prime}}^{\dagger}(t)\right| 0\right\rangle$. Thereby we neglect the two-particle terms which are of the structure $c_{\alpha s}^{\dagger} c_{\beta s}^{\dagger}|0\rangle\langle 0| c_{\beta s} c_{\alpha s}$. Formally, these terms drop out in the limit of strong Coulomb repulsion because they are accompanied by a rapidly oscillating phase factor $\exp \left(-\mathrm{i} U N_{\text {wire }} \tau / \hbar\right)$. Then the $\tau$ integration results in a factor $f_{\mathrm{L}}\left(\epsilon_{\alpha, k}+U\right)$ which vanishes in the limit of large $U$. Since the total Hamiltonian (1) is diagonal in the spin index $s$, we find that the density matrix elements $\rho_{\alpha s, \beta s^{\prime}}$ are spin-independent as well so that after a transient stage

$$
\rho_{\alpha \uparrow, \beta \uparrow}(t)=\rho_{\alpha \downarrow, \beta \downarrow}(t) \equiv \rho_{\alpha \beta}(t)
$$

and $\rho_{\alpha \uparrow, \beta \downarrow}=0$. Moreover, at large times, the density operator (19) will acquire the time periodicity of the driving field [8] and, thus, can be decomposed into the Fourier series

$$
\rho_{\alpha \beta}(t)=\sum_{k} \mathrm{e}^{-\mathrm{i} k \Omega t} \rho_{\alpha \beta, k}
$$

and $\rho_{00}(t)$ accordingly.

After some algebra, we arrive at a set of $N^{2}$ coupled equations of motion for $\rho_{\alpha \beta}(t)$ which in Fourier represen- tation read

$$
\begin{aligned}
& \mathrm{i}\left(\epsilon_{\alpha}-\epsilon_{\beta}-k \hbar \Omega\right) \rho_{\alpha \beta, k} \\
& =\frac{\Gamma_{\mathrm{L}}}{2} \sum_{k^{\prime}, k^{\prime \prime}}\left\langle\varphi_{\alpha, k^{\prime}+k^{\prime \prime}} \mid 1\right\rangle\left\langle 1 \mid \varphi_{\beta, k+k^{\prime \prime}}\right\rangle \rho_{00, k^{\prime}} \\
& \times\left(f_{\mathrm{L}}\left(\epsilon_{\alpha, k^{\prime}+k^{\prime \prime}}\right)+f_{\mathrm{L}}\left(\epsilon_{\beta, k+k^{\prime \prime}}\right)\right) \\
& -\frac{\Gamma_{\mathrm{L}}}{2} \sum_{\alpha^{\prime}, k^{\prime}, k^{\prime \prime}}\left\langle\varphi_{\alpha, k^{\prime}+k^{\prime \prime}} \mid 1\right\rangle\left\langle 1 \mid \varphi_{\alpha^{\prime}, k+k^{\prime \prime}}\right\rangle \rho_{\alpha^{\prime} \beta, k^{\prime}} \bar{f}_{\mathrm{L}}\left(\epsilon_{\alpha^{\prime}, k+k^{\prime \prime}}\right) \\
& -\frac{\Gamma_{\mathrm{L}}}{2} \sum_{\beta^{\prime}, k^{\prime}, k^{\prime \prime}}\left\langle\varphi_{\beta^{\prime}, k^{\prime}+k^{\prime \prime}} \mid 1\right\rangle\left\langle 1 \mid \varphi_{\beta, k+k^{\prime \prime}}\right\rangle \rho_{\alpha \beta^{\prime}, k^{\prime}} \bar{f}_{\mathrm{L}}\left(\epsilon_{\beta^{\prime}, k^{\prime}+k^{\prime \prime}}\right) \\
& + \text { same terms with the replacement } 1, \mathrm{~L} \rightarrow N, \mathrm{R} \text {. }
\end{aligned}
$$

In order to solve these equations, we have to eliminate $\rho_{00, k}$ which is most conveniently done by inserting the Fourier representation of the normalisation condition

$$
\operatorname{tr} \rho_{\text {wire }}(t)=\rho_{00}(t)+2 \sum_{\alpha} \rho_{\alpha \alpha}(t)=1
$$

In order to obtain for the current an expression that is consistent with the restriction to one excess electron, we compute the expectation values in the current formula (9) with the reduced density operator (19) and insert the Floquet representation (15) of the wire operators. Performing an average over the driving period, we obtain for the dc current the expression

$$
\begin{aligned}
I=\frac{2 e \Gamma_{\mathrm{L}}}{\hbar} \operatorname{Re} \sum_{\alpha, k} & \left(\sum_{\beta, k^{\prime}}\left\langle\varphi_{\beta, k^{\prime}+k} \mid 1\right\rangle\left\langle 1 \mid \varphi_{\alpha, k}\right\rangle \rho_{\alpha \beta, k^{\prime}} \bar{f}_{\mathrm{L}}\left(\epsilon_{\alpha, k}\right)\right. \\
& \left.-\sum_{k^{\prime}}\left\langle\varphi_{\alpha, k^{\prime}+k} \mid 1\right\rangle\left\langle 1 \mid \varphi_{\alpha, k}\right\rangle \rho_{00, k^{\prime}} f_{\mathrm{L}}\left(\epsilon_{\alpha, k}\right)\right) .
\end{aligned}
$$

Physically, the second contribution of the current formula (24) describes the tunnelling of an electron from the left lead to the wire and, thus, is proportional to $\rho_{00} f_{\mathrm{L}}$ which denotes the probability that a lead state is occupied while the wire is empty. The first terms corresponds to the reversed process namely the tunnelling on an electron from site $|1\rangle$ to the left lead.

The results of this section allow us the numerical computation of the dc current through a driven conductor in the the following way: First, we solve the quasienergy equation (12) which provides the coefficients $\left\langle\varphi_{\alpha, k} \mid n\right\rangle$. Next, we solve the master equation (22) and insert the solution into the current formula (24).

\section{Separating interaction and spin}

In order to determine the role of a strong interaction, we shall compare below our results to the non-interacting case. Moreover, a particular consequence of strong Coulomb repulsion is the mutual blocking of different spin channels. This motivates us to also compare to the case of spinless electrons which is physically realised by spin polarisation. In this section, we adapt our master equation approach to these situations. 
Franz J. Kaiser et al.: Coulomb repulsion effects in driven electron transport

\subsection{Spinless electrons}

In order to describe spinless electrons, we drop in the initial Hamiltonian all spin indices. Physically, this limit is realised by a sufficiently strong magnetic field that polarises all electrons contributing to the transport. By the same calculation as in Section 3, we then obtain for the current also the expression (24) but without the prefactor 2 . The factor 2 is also no longer present in the normalisation condition (23) which now reads

$$
\operatorname{tr} \rho_{\text {wire }}(t)=\rho_{00}(t)+\sum_{\alpha} \rho_{\alpha \alpha}(t)=1 .
$$

\subsection{Non-interacting electrons}

In the absence of interactions, $U=0$, each spin degree of freedom can be treated separately. Still one has to consider for each spin projection up to $N$ electrons so that the relevant Hilbert space has the dimension $2^{N}$. Therefore, it is more efficient to consider the single-particle density matrix

$$
R_{\alpha \beta}(t)=\left\langle c_{\alpha s}^{\dagger}(t) c_{\beta s}(t)\right\rangle_{t}=R_{\beta \alpha}^{*}(t)=\sum_{k} \mathrm{e}^{-\mathrm{i} k \Omega t} R_{\alpha \beta, k},
$$

which is of dimension $N^{2}$ and nevertheless contains all relevant information. The Fourier decomposition in the last expression uses the fact that, at asymptotically large times, $R_{\alpha \beta}(t)$ becomes time-periodic.

We express the time derivative of $R_{\alpha \beta}(t)$ with the master equation (8) and insert for $\tilde{c}_{\alpha}(t, t-\tau)$ the Floquet representation (18). After some algebra, we obtain for the Fourier coefficients $R_{\alpha \beta, k}$ the equation

$$
\begin{aligned}
& \mathrm{i}\left(\epsilon_{\alpha}-\epsilon_{\beta}+k \hbar \Omega\right) R_{\alpha \beta, k} \\
&=\frac{\Gamma_{\mathrm{L}}}{2} \sum_{k^{\prime}}\left(\sum_{\beta^{\prime}, k^{\prime \prime}}\left\langle\varphi_{\beta, k^{\prime}+k^{\prime \prime}} \mid 1\right\rangle\left\langle 1 \mid \varphi_{\beta^{\prime}, k+k^{\prime \prime}}\right\rangle R_{\alpha \beta^{\prime}, k^{\prime}}\right. \\
&+\sum_{\alpha^{\prime}, k^{\prime \prime}}\left\langle\varphi_{\alpha^{\prime}, k^{\prime}+k^{\prime \prime}} \mid 1\right\rangle\left\langle 1 \mid \varphi_{\alpha, k+k^{\prime \prime}}\right\rangle R_{\alpha^{\prime} \beta, k^{\prime}} \\
&-\left\langle\varphi_{\beta, k^{\prime}-k} \mid 1\right\rangle\left\langle 1 \mid \varphi_{\alpha, k^{\prime}}\right\rangle f_{\mathrm{L}}\left(\epsilon_{\alpha, k^{\prime}}\right) \\
&\left.-\left\langle\varphi_{\beta, k^{\prime}} \mid 1\right\rangle\left\langle 1 \mid \varphi_{\alpha, k^{\prime}+k}\right\rangle f_{\mathrm{L}}\left(\epsilon_{\beta, k^{\prime}}\right)\right) \\
&+ \text { terms with the replacement } \mathrm{L}, 1 \rightarrow \mathrm{R}, N .
\end{aligned}
$$

In contrast to the master equation (22) for the limit of strong Coulomb repulsion, no blocking factors $1-f_{\mathrm{L} / \mathrm{R}}$ emerge. This is characteristic for the non-interacting limit and is also found in the exact scattering formula [8].

Using the single-particle density matrix $R_{\alpha \beta}$, one can evaluate the current expectation value (9). Thereby the spin degree of freedom enters simply as a factor 2 so that we obtain for the current per spin the expression

$$
\begin{aligned}
I=\frac{e \Gamma_{\mathrm{L}}}{\hbar} \sum_{\alpha, k}[ & \operatorname{Re} \sum_{\beta, k^{\prime}}\left\langle\varphi_{\beta, k^{\prime}+k} \mid 1\right\rangle\left\langle 1 \mid \varphi_{\alpha, k}\right\rangle R_{\alpha \beta, k^{\prime}} \\
& \left.-\left|\left\langle 1 \mid \varphi_{\alpha, k}\right\rangle\right|^{2} f_{\mathrm{L}}\left(\epsilon_{\alpha, k}\right)\right] .
\end{aligned}
$$

For a detailed derivation see Ref. [36].

\section{Interplay of dipole radiation and Coulomb repulsion}

In our model Hamiltonian (1) we have already specified the interaction, the lead Hamiltonian, and the wire-lead coupling. By contrast, for the Hamiltonian of the driven wire, we have thus far only assumed that the external field is periodic in time. In the following we focus on models where the single-particle dynamics is determined by the $N$-site tight-binding Hamiltonian

$$
\begin{aligned}
H_{n n^{\prime}}(t)= & -\Delta \sum_{n=1}^{N-1}(|n\rangle\langle n+1|+| n+1\rangle\langle n|) \\
& +\sum_{n=1}^{N}\left\{E_{n}+A x_{n} \cos (\Omega t)\right\}|n\rangle\langle n| .
\end{aligned}
$$

Neighbouring sites are coupled by a tunnel matrix element $\Delta$. The onsite energies $E_{n}$ are modulated by a harmonically time-dependent dipole force, where the amplitude $A$ is given by the electrical field amplitude multiplied by the electron charge and the distance between neighbouring sites. $x_{n}=\frac{1}{2}(N+1-2 n)$ denotes the scaled position of site $|n\rangle$. Depending on the onsite energies $E_{n}$, one observes various phenomena which we discuss in the following.

\subsection{Resonant excitations of bridged molecular wires}

A frequently studied model is the so-called bridged molecular wire [42] sketched in Fig. 1. It consists of a molecule with $N$ sites, where the first and the last site - the donor $|1\rangle$ and the acceptor $|N\rangle$ - are connected to respective leads. The energies of these two sites are assumed to be close to the chemical potentials of the respective leads, $\mu_{\mathrm{L}} \lesssim E_{1}=E_{N} \lesssim \mu_{\mathrm{R}}$. The remaining $N-2$ orbitals lie well above the chemical potentials at an energy $E_{\mathrm{B}} \gg \Delta$ which defines the bridge height. In the presence of laser excitations, we expect an enhanced current whenever the energy quanta of the laser field $\hbar \Omega$ matches the energy difference between donor/acceptor and one of the $N-2$ bridge levels. This photon-assisted tunnelling indeed exhibits resonance peaks which obey the scaling law $I_{\text {peak }} \propto A^{2} /(N-1) \Gamma[8]$.

Figure 2 shows the dc current as a function of the laser frequency for the three approaches considered herein. In all these cases, the resonance peaks are at the same frequencies. As a further interesting feature, we find that in the strongly interacting case $U=\infty$, the current does not depend on whether one includes the spin degree of freedom. This behaviour can be understood qualitatively in the following way: If there is no excess electron on the wire, an electron can enter from both the "spin-up channel" and the "spin-down channel". By contrast, when leaving the wire, the electron spin is preserved so that only either channel is available. This means that including the electron spin effectively modifies the in-tunnelling rates by 


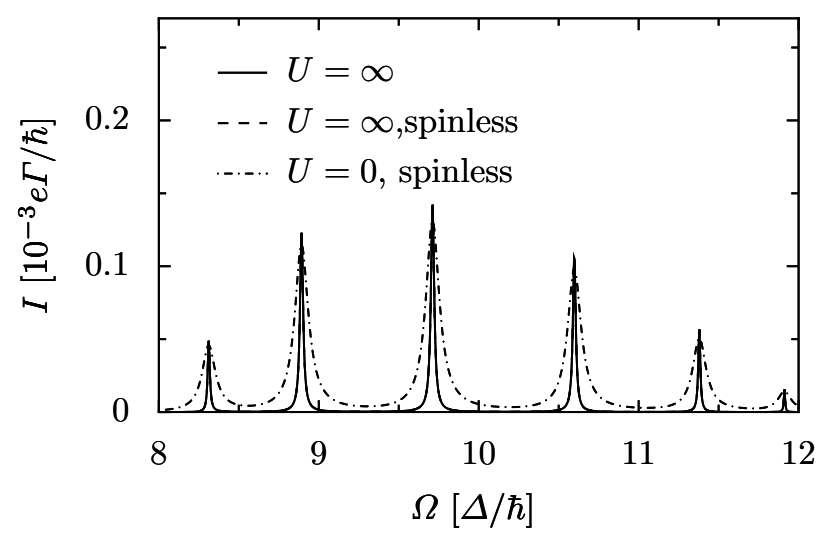

Fig. 2. Photon-assisted current in a bridged molecular wire with length $N=8$ and height $E_{\mathrm{B}}=10 \Delta$. Leads with a chemical potential difference $e V=5 \Delta$ couple to the wire with an effective strength $\Gamma=0.1 \Delta$. The driving amplitude is $A=0.3 \Delta$. For $U=\infty$, the current for spinless electrons coincides with the one for real electrons.

a factor 2. The electron dynamics within the wire, however, is described by the coherent first term of the master equation (8) which is spin-independent. Consequently, we expect that the spin only plays a minor role whenever the tunnelling from the donor to the acceptor becomes the bottleneck for the electrons. This is indeed the case for the transport across a barrier considered here.

As compared to the non-interacting case, one notices that strong Coulomb repulsion modifies the shape of the peaks: They become slightly higher and much sharper. This effect is more pronounced for large wires. A closer look at the resonance peaks for wires with up to $N=10$ sites (not shown) indicates that the resonance widths scale roughly like $1 / N$. This is possibly caused by the fact that the Coulomb repulsion reduces the number of available (many-particle) wire states and, thus, the number of decay channels. This relates to the observation made in Ref. [41], namely that Coulomb repulsion can improve quantum coherence and thereby enhance the current.

Since quantum coherence is also temperature dependent, it is natural to ask whether the resonance peaks become sharper for lower temperatures. Figure 3 shows the width of the central peak of Fig. 2 as a function of the temperature. While in the non-interacting case, the peak widths are essentially temperature independent, the situation changes for strong Coulomb repulsion: There one finds that with an increasing temperature, the peaks become roughly twice as broad once the temperature exceeds $T=\Delta / k_{\mathrm{B}}$. We attribute this behaviour to the reduced coherence for thermal energies that are larger than the tunnelling matrix element.

\subsection{Non-adiabatic electron pumping}

Another well studied phenomenon in driven transport is coherent electron pumping, i.e., the creation of a nonvanishing dc current by ac fields in the absence of any net bias. For adiabatically slow driving, this effect exists only

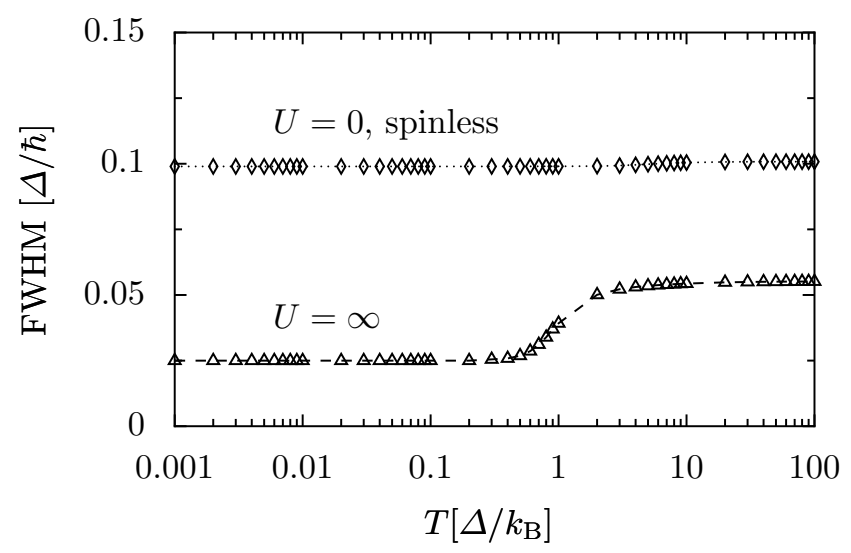

Fig. 3. Temperature dependence of the widths of the resonance peaks at $\Omega \approx 9.71 \Delta / \hbar$. The graph shows the full widths at half of the maximum. All other parameters are as in Fig. 2. For $U=\infty$, the values for spinless electrons and real electrons coincide.

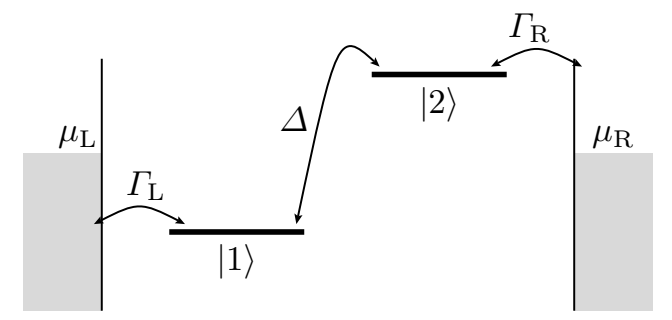

Fig. 4. Tight-binding model for two coupled quantum dots in pump configuration, i.e. in the absence of a bias voltage but with an internal bias $E_{2}-E_{1} \neq 0$ which breaks reflection symmetry.

in the absence of time-reversal symmetry [18-20]. Beyond the adiabatic regime, this is no longer the case: For fast, time-periodic driving fields, it can be shown that the relevant symmetry is the so-called generalized parity which is defined as the invariance under spatial reflection in combination with a time shift by half a driving period [8]. Non-adiabatic electron pumping is particularly interesting because at internal resonances of the central system the pump current can assume rather large values [10,22, 35], while at the same time the current noise is remarkably low $[24]$.

For studying the influence of strong Coulomb repulsion on non-adiabatic electron pumping, we consider the setup sketched in Fig. 4. Of particular interest is the parameter regime with large internal bias and intermediate dot-lead coupling because in this regime, the current-to-noise ratio is most favourable [24]. The currents obtained for the three considered approaches are shown in Fig. 5. Again we find that the spin degree of freedom is not of major influence, which indicates that the transport is governed by internal excitations; cf. the discussion in the preceeding subsection.

The influence of the Coulomb repulsion is a modification of the current peak height up to $5 \%$. This means that interactions are here much less important than for photonassisted transport: The reason for this is that for our pump configuration, one energy level lies below the Fermi energy 


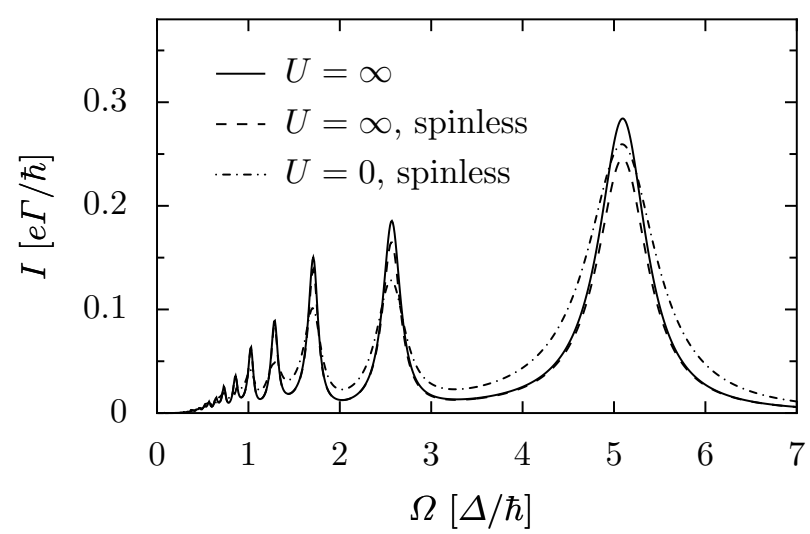

Fig. 5. Pump current for strong Coulomb repulsion and for non-interacting electrons as a function of the frequency. The dot levels with energies $E_{1}=-2.5 \Delta$ and $E_{2}=2.5 \Delta$ are couple to the leads with strength $\Gamma=0.3 \Delta$. The driving amplitude is $A=3.7 \Delta$ and the temperature $k_{\mathrm{B}} T=0.005 \Delta$.

while the other lies well above. Thus in equilibrium for a sufficiently small dot-lead coupling, the left site is occupied while the right site is empty, whatever the interaction strength. Thus, the double dot is populated with only one electron so that interactions become irrelevant. Unless the driving amplitude is huge, this occupation is altered only slightly. Consequently interactions do not modify the current significantly. We emphasise that for strong dot-lead coupling $\Gamma$ and finite interaction $U$, these arguments no longer hold true.

\subsection{Coherent current control}

An intriguing example of quantum control is the so-called coherent destruction of tunnelling in a double-well potential by a suitable driving field [43], which can be explained within a rotating-wave approximation: In the driving dominated regime, the tunnel matrix element is essentially replaced by an effective tunnel matrix element $\Delta_{\text {eff }}=J_{0}(A / \hbar \Omega)$, where $J_{0}$ is the zeroth order Bessel function of the first kind and $A$ the driving amplitude $[44,45]$. Related effects have been predicted for driven tight-binding lattices [46]. For driven transport between two leads, the corresponding situation has been investigated only recently: It has been found that driving fields that suppress tunnelling in a closed driven system, also suppress the current through the corresponding open system $[14,47]$.

A setup in which this coherent current suppression can be studied is sketched in Fig. 6. It is characterized by the fact that all internal levels lie within the voltage window, i.e. below one chemical potential and above the other. For this system, strong interaction has already a significant influence on the current in the absence of any driving field [41]: For $U=0$, the system is half filled, which means it is populated by $N / 2$ electron, and the current is independent of $N$. By contrast for $U=\infty$, the stationary population is $N /(N+1)$ and the current becomes $I \propto 1 /(N+1)$.

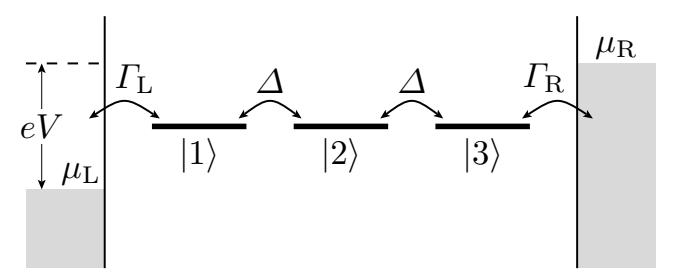

Fig. 6. Triple quantum dot configuration for coherent current control: A large bias voltage and the unbiased dot levels with $E_{n}=0$ ensure optimal transport in the absence of the driving.

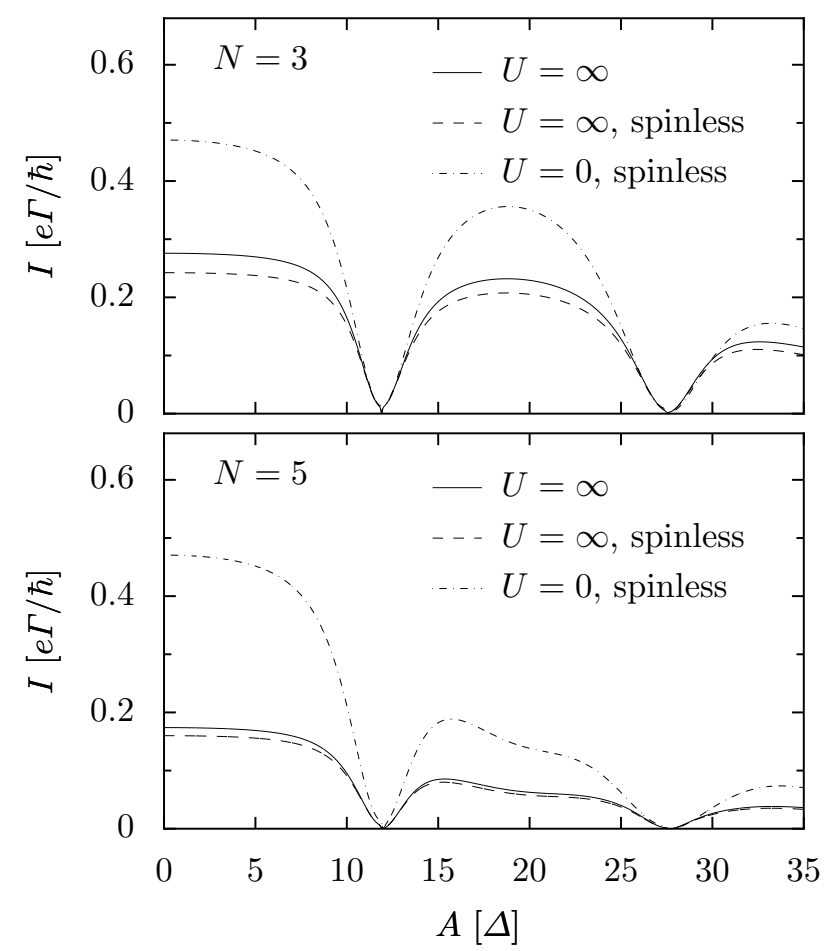

Fig. 7. Coherent current suppression as a function of the driving amplitude $A$ for a wire that consists of $N=3$ sites. The applied voltage is $e V=50 \Delta$, the driving frequency $\Omega=5 \Delta$, and the wire-lead coupling $\Gamma=0.5 \Delta$.

Figure 7 shows the influence of Coulomb repulsion on the current suppression studied in Ref. [17]. Independent of the interaction, one finds that the current almost vanishes whenever the ratio $A / \hbar \Omega$ matches a zero of the Bessel function $J_{0}$. If the driving amplitude is far from the values for which the current is suppressed, we observe the behaviour found for the static situation, namely that Coulomb repulsion reduces the current by a factor $1 /(N+1)$ for spinless electrons [41]. If one considers the spin, this factor becomes $2 /(2 N+1)$. In the vicinity of the current suppressions, by contrast, the influence of both the inclusion of the spin and the interaction is less pronounced. In this regime, the effective tunnel matrix element $\Delta_{\text {eff }}$ is small, so that tunnelling along the wire happens at a low rate. This again indicates that whenever the transport is limited by the dynamics within the wire, the influence of interaction is rather small. 
Franz J. Kaiser et al.: Coulomb repulsion effects in driven electron transport

\section{Conclusions}

We studied the influence of strong interaction on the transport properties of ac-driven coherent conductors. In particular, we compared the strongly interacting case with the opposite extreme of non-interacting electrons. Moreover, we worked out the relevance of the spin degree of freedom for weak wire-lead coupling. In our studies, we considered three archetypical effects, namely photon-assisted tunnelling through bridged molecular wires, non-adiabatic electron pumping, and coherent current suppression.

The most significant effect is found for photon-assisted tunnelling where Coulomb repulsion renders the resonance linewidths much sharper. Thus unfortunately, interactions might contribute to the difficulties in photon-assited tunnelling experiments with molecular wires. By contrast, Coulomb repulsion is not too relevant for electron pumping in double quantum dots. For coherent current suppression, the same holds true only for parameters for which the current is already significantly reduced. Outside this region, one finds that Coulomb repulsion reduces the current essentially in the same way as in the absence of driving.

The two extreme cases of zero and very strong interaction do not necessarily allow a simple interpolation. Thus, it is desireable to extend the present studies to finite values of the interaction strength, which requires the generalisation of our formalism to at least a second excess electron. Moreover, the dc current is certainly not the only relevant quantity for the characterisation of the electron transport. Investigating the influence of Coulomb repulsion on, e.g., the current noise would complement the picture drawn above.

We thank M. Strass and A. Nitzan for interesting discussions. This work has been supported by Deutsche Forschungsgemeinschaft through SFB 484 and SPP 1243. One of us (FJK) acknowledges funding by Bayerisches Staatsministerium für Wissenschaft, Forschung und Kunst through Elitenetzwerk Bayern.

\section{References}

1. M. A. Reed, C. Zhou, C. J. Muller, T. P. Burgin, and J. M. Tour, Science 278, 252 (1997).

2. X. D. Cui et al., Science 294, 571 (2001).

3. J. Reichert, R. Ochs, D. Beckmann, H. B. Weber, M. Mayor, and H. von Löhneysen, Phys. Rev. Lett. 88, 176804 (2002).

4. J. Reichert, H. B. Weber, M. Mayor, and H. von Löhneysen, Appl. Phys. Lett. 82, 4137 (2003).

5. S. Kohler, J. Lehmann, S. Camalet, and P. Hänggi, Israel J. Chem. 42, 135 (2002).

6. A. Keller, O. Atabek, M. Ratner, and V. Mujica, J. Phys. B 35, 4981 (2002).

7. A. Tikhonov, R. D. Coalson, and Y. Dahnovsky, J. Chem. Phys. 117, 567 (2002).

8. S. Kohler, J. Lehmann, and P. Hänggi, Phys. Rep. 406, 379 (2005).
9. R. H. Blick, R. J. Haug, J. Weis, D. Pfannkuche, K. von Klitzing, and K. Eberl, Phys. Rev. B 53, 7899 (1996).

10. W. G. van der Wiel, S. De Franceschi, J. M. Elzerman, T. Fujisawa, S. Tarucha, and L. P. Kouwenhoven, Rev. Mod. Phys. 75, 1 (2003).

11. T. H. Oosterkamp et al., Nature 395, 873 (1998).

12. W. G. van der Wiel, T. Fujisawa, T. H. Oosterkamp, and L. P. Kouwenhoven, Physica B (Amsterdam) 272, 31 (1999).

13. G. Platero and R. Aguado, Phys. Rep. 395, 1 (2004).

14. J. Lehmann, S. Camalet, S. Kohler, and P. Hänggi, Chem. Phys. Lett. 368, 282 (2003).

15. F. Grossmann, Phys. Rev. B 70, 113306 (2004).

16. S. Welack, M. Schreiber, and U. Kleinekathöfer, J. Chem. Phys. 124, 044712 (2006).

17. S. Camalet, J. Lehmann, S. Kohler, and P. Hänggi, Phys. Rev. Lett. 90, 210602 (2003); S. Camalet, et al., Phys. Rev. B 70, 155326 (2004).

18. P. W. Brouwer, Phys. Rev. B 58, R10135 (1998).

19. B. L. Altshuler and L. I. Glazman, Science 283, 1864 (1999).

20. M. Switkes, C. M. Marcus, K. Campman, and A. C. Gossard, Science 283, 1905 (1999).

21. B. Wang, J. Wang, and H. Guo, Phys. Rev. B 65, 073306 (2002).

22. C. A. Stafford and N. S. Wingreen, Phys. Rev. Lett. 76, 1916 (1996).

23. M. Wagner and F. Sols, Phys. Rev. Lett. 83, 4377 (1999).

24. M. Strass, P. Hänggi, and S. Kohler, Phys. Rev. Lett. 95, 130601 (2005).

25. J. H. Shirley, Phys. Rev. 138, B979 (1965).

26. S. Kohler, T. Dittrich, and P. Hänggi, Phys. Rev. E 55, 300 (1997).

27. M. Grifoni and P. Hänggi, Phys. Rep. 304, 229 (1998).

28. M. Moskalets and M. Büttiker, Phys. Rev. B 66, 205320 (2002).

29. J. Lehmann, S. Kohler, P. Hänggi, and A. Nitzan, Phys. Rev. Lett. 88, 228305 (2002).

30. C. Bruder and H. Schoeller, Phys. Rev. Lett. 72, 1076 (1994).

31. J. Lehmann, S. Kohler, V. May, and P. Hänggi, J. Chem. Phys. 121, 2278 (2004).

32. U. Kleinekathöfer, G. Li, S. Welack, and M. Schreiber, Europhys. Lett. 75, 139 (2006).

33. Yu. V. Nazarov, Physica B 189, 57 (1993).

34. S. A. Gurvitz and Ya. S. Prager, Phys. Rev. B 53, 15932 (1996).

35. P. Brune, C. Bruder, and H. Schoeller, Phys. Rev. B 56, 4730 (1997).

36. J. Lehmann, S. Kohler, P. Hänggi, and A. Nitzan, J. Chem. Phys. 118, 3283 (2003).

37. T. Novotný, Europhys. Lett. 59, 648 (2002).

38. E. G. Petrov and P. Hänggi, Phys. Rev. Lett. 86, 2862 (2001).

39. E. G. Petrov, V. May, and P. Hänggi, Chem. Phys. 281, 211 (2002).

40. J. Lehmann, G.-L. Ingold, and P. Hänggi, Chem. Phys. 281, 199 (2002).

41. F. J. Kaiser, M. Strass, S. Kohler, and P. Hänggi, Chem. Phys. 322, 193 (2006).

42. A. Nitzan, Annu. Rev. Phys. Chem. 52, 681 (2001).

43. F. Grossmann, T. Dittrich, P. Jung, and P. Hänggi, Phys. Rev. Lett. 67, 516 (1991). 
44. F. Großmann, P. Jung, T. Dittrich, and P. Hänggi, Z. Phys. B 84, 315 (1991).

45. F. Großmann and P. Hänggi, Europhys. Lett. 18, 571 (1992).

46. M. Holthaus, Phys. Rev. Lett. 69, 351 (1992).

47. S. Kohler, S. Camalet, M. Strass, J. Lehmann, G.-L. Ingold, and P. Hänggi, Chem. Phys. 296, 243 (2004). 